\title{
The Role of Telemedicine using computer communication network, Internet and Healthcare Management support in 2014 India
}

\author{
S.saravanan ${ }^{1}$, Dr. S. Sundaramurthy Reddy ${ }^{2}$ \\ Asst.Professor (SG), CSE Department, Rajiv Gandhi college Engineering and Technology, Puducherry, India ${ }^{1}$ \\ Professor, MBA Department, Rajiv Gandhi college Engineering and Technology, Puducherry, India ${ }^{2}$
}

\begin{abstract}
All the small scale hospitals can be implemented telemedicine service using $3 \mathrm{G}$ internet, computer communication and mobile network In future need emergency treatment for critical position of accident patient. This paper explains the role of Emergency Telemedicine service using internet, computer communication with Healthcare management. The ISRO supports Telemedicine service using $3 \mathrm{G}$ internet in rural area and development of telemedicine service fulfilment $95 \%$ using computer communication network. In development of $22 \%$ in Telemedicine service in 2000 using $2 \mathrm{G}$ internet service developed up to $95 \%$ by using $3 \mathrm{G}$ internet in 2014
\end{abstract}

Keywords: Telemedicine service, 3G internet, computer and mobile communication network , Information Technology, Care Transformation, Performance Improvement, Strategy

\section{INTRODUCTION}

India is a vast country with more than 1 billion population occupying an area of 3 million sq $\mathrm{km}$. It consists of 29 states and 6 Union Territories governed by a federal system. There is no national health insurance policy for the country. Government supported healthcare delivery follows a three tier system and is the primary responsibility of each state. It has been observed that there is a great deal of disparity in quality and access to healthcare between urban and rural regions. This healthcare divide needs to be bridged since most of the Indian population live in rural areas. Telemedicine is an emerging Information and Communication enabled health technology which has the potential to facilitate access to healthcare in underprivileged population if absorbed into existing healthcare delivery system. The scale of e-health services in India has been very small so far considering it's size, mostly limited to medical transcription, health awareness through portals, telemedicine, hospital management system and customer service using the internet. Both government and private agencies are venturing into Tele-healthcare by providing hardware and software solutions.

Tele-health industry is coming up and policy and standard issues are getting addressed. Government efforts are directed towards setting up standards and defining IT enabled healthcare infrastructure in the country. Recently in the year 2005 the Ministry of Health Government of India has constituted a National Task Force on Telemedicine. Human resource development in e health field is also realized in 2014 Health government of India using $3 \mathrm{G}$ internet to telemedicine service to rural area [1 to 10].

\section{The Role of TELEMEdicine HEALTHCARE MANAGEMENT}

A. Leadership and governance structure supports your organization, and more specifically, our care management infrastructure

. Our CEO and his leadership team have had the vision and courage to move forward with population health management in the face of some pretty daunting barriers, including lack of payment reform supporting this new care delivery. It is this kind of leadership that challenges the organization to reach for a higher goal that leads to success. This is not without risk, but without risk there can be no reward. As far as governance, we have a very integrated model of care with employed physicians and a paired physician-administrative leadership model. We have about 1,800 integrated employed physicians across the health system as well as roughly 800 NPs. Case and care management leadership has the same importance in our organization as our physician leadership, our clinics, our finance leadership, our materials management leadership, etc. We view case and care management on par with all of our clinical and operational departments.

\section{B. The most critical foundational elements for an organization embarking on population health management.}

For health systems seeing downward pressure on margins, fewer admissions, and an increased cost of delivering care, there are a few baseline factors they might consider. First, ensuring the system has a strong primary care base is fundamental to both capturing the patient population and 
subsequently managing their care. This network needs to solutions because they see technology playing a major role have a fully integrated EMR and the analytics in other industries. Technology itself is also not a barrier, infrastructure in place to take data and turn it into as it is advancing rapidly for reasons other than health knowledge. Expanding on this foundation, health systems care. Health providers have had the opportunity to learn should build a more robust access model at the primary from and build upon those developments to enhance the care level—a system's entry to care. This model should patient experience. Workflow processes, however, have include extensions of primary care (i.e. convenient care, been much more interesting and challenging. When you go walk-in clinics, worksite clinics) and build a team to a physician's office, the schedule might be running approach to care delivery based on Patient Centered behind by an hour, but you know your PCP is there. There Medical Home attributes, including clinic-based care is a level of uncertainty when working with telemedicine; managers. These care managers can offload tasks from if a patient has a telemedicine visit in a rural town in physicians and NPs, thus increasing patient volumes in an Oklahoma, will the workflow be efficient enough to appropriate manner while improving patient care at the ensure there will be a physician on the other side of that same time. Mercy chose to leverage telemedicine to television screen? And on time? I think workflow further expand access by implementing e-Consultation and processes, while not a true barrier, will pose as more of a e-Visit functionality. Such investments are all part of our challenge than technology or user acceptance.

commitment to developing our Virtual Care Centre a hub for health technology advancements and patient-centric service innovation-developed under our Centre for Innovative Care. Telemedicine is an efficient and effective way to expand patient access and volume

\section{Telemedicine fit into the system's broader care coordination strategy.}

Telemedicine service in four areas:

- $\quad$ Monitoring: e-ICU, tele-sepsis, and e-SNF initiatives

- $\quad$ Face-to-face consultation and consultation ondemand: tele-stroke, tele-psychiatry, other tele-specialties, and ancillary services

- $\quad$ Store and forward: radiology, imaging, dermatology, follow-up visits, or minor acuity visits

- $\quad$ Asynchronous care: e-visits, internet communication, and social media use

\section{E. Health's telemedicine and clinical initiatives help meet the long-term objectives of high-quality, longitudinal care delivery.}

The goal is to coordinate the continuum of care so patients get the right care effectively and efficiently. We are confident that this can be achieved with the initiatives we have been pursuing: team-based care, telemedicine via our Virtual Care Center, and strategic collaboration across systems. It is our hope and vision that we can take the infrastructure and knowledge base that we have developed and collaborate with other progressive and likeminded health systems. This way, there will be one Virtual Care Center that then provides all patients health care access rather than having 50 independent virtual care centers. This is a modality that definitely benefits from scale and can lower everybody's costs, while at the same time providing more effective and efficient care with exceptional service.

We have been restructuring our physician model to be more team-based, allowing for better care coordination. Parallel to this shift in our staffing model, we are developing our telemedicine infrastructure to use these care teams more efficiently. Telemedicine allows us to distribute our team-care services without necessarily moving those providers between sites. For example, patients can have face-to-face consultations with providers through a television monitor. This asynchronous care model also uses physician time more efficiently because much of patients' data can be gathered from home monitoring, uploaded to the EMR, and aggregated for providers to access. Our tele-sepsis model has more than halved the traditional mortality with sepsis and cut LOS by $50 \%$.

\section{The main challenges in implementing and expanding telemedicine services.}

Interestingly, they were not what I had initially anticipated. I had thought patient acceptance would be a roadblock, but patients are in fact asking for IT-based

\section{F. Steps are taking to ensure a streamlined patient experience at Mercy Health.}

The health care delivery model has historically been provider-centric and siloed. With the advent of the EHR, improved technology, and a national culture gearing to internet socialization, our basic care model is no longer effective. To this end, we are shifting to a patient-centered delivery model that provides care where the patients are and uses modalities that patients have become accustomed to.Currently, we have every piece of the telemedicine continuum in place. Now it's about ensuring we get the right care to patients at the right time. Our health care system does not suffer from lack of clinical ability and knowledge - we don't need new ways to take care of congestive heart failure. The priority is identifying patients in need and providing them access to the best care possible. Overall, it is just a matter of resources, time, and leadership. It doesn't happen overnight, but it is the Queen Mary and not the Titanic-transforming to a new care model takes time, but with the right leadership and vision you can avoid the icebergs. 
G. kind of central staff do I need for a live, interactive or hybrid telemedicine consultation service.

Critical to the success of a telemedicine consultation service is a staff of knowledgeable and skilled individuals who can establish and operate the service. This group must be of sufficient size to successfully carry out a variety of tasks including:

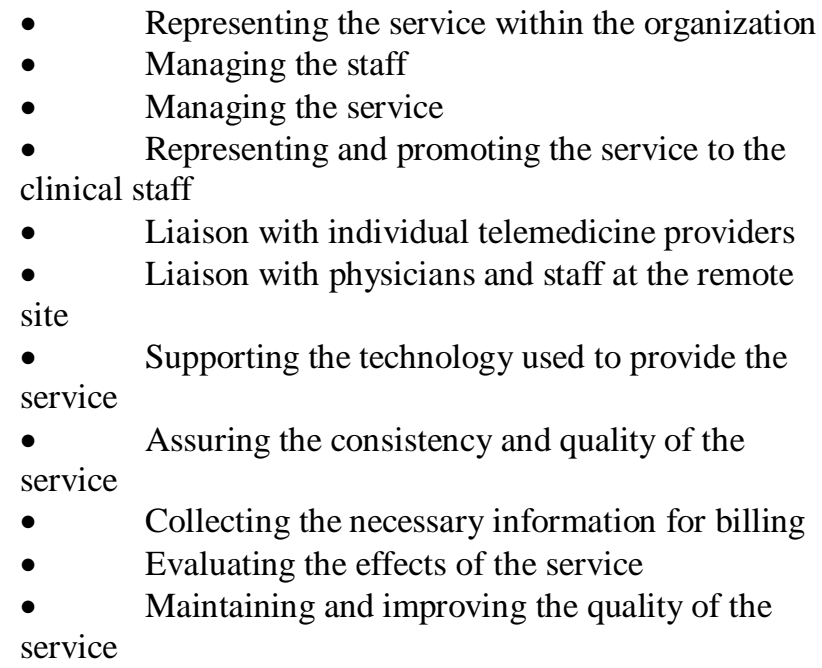

The group must be able to work together as a cohesive team with each member contributing his or her unique knowledge and skills in order to create and operate a successful service. Though there are several roles that need to be filled, one person may take on more than one role.

\section{H. The role of the Project Manager}

The project manager is the leader of the telemedicine team who represents the group and advocates for it to the organization and manages the staff. The manager reports on the function and progress of the service, negotiates budgetary issues, recruits and hires staff, sets priorities in consultation with the team and supervises its functioning. Qualifications for the project manager position include a deep understanding of telemedicine consultation services, previous project management experience, good working relationships with physicians, personnel management skills and an understanding of the business needs of the organization.

\section{The role of the Medical/Clinical Director}

The medical director plays an important role on the team as the liaison with the organization's medical staff. The medical director helps to provide clinical credibility for the program, representing the clinical staff and recruiting providers for the service. This person also provides practical clinical advice on creation and operation of the service. Qualifications usually include an enthusiasm for telemedicine, knowledge of the benefits of the service, ability to convey to other physicians the experience of practicing via telemedicine and a relatively senior standing among the clinical staff.

\section{J. The role of the Clinical Coordinator}

The clinical coordinator has responsibility for all clinical aspects of the services provided. This includes training of providers and any clinical support staff, coordinating the scheduling of patients and providers, developing operational protocols, and directing any process and improvement activities. Qualifications for the clinical coordinator include clinical experience, previous experience with telemedicine services (if possible), skills in training others, and knowledge for conducting quality improvement projects

\section{$K$. The role of the Technology Coordinator}

Live, interactive and hybrid telemedicine sessions depend on the functioning of a complex set of technologies. These technologies range from local computer systems to videoconferencing devices (e.g., microphones, video cameras), ancillary devices (e.g., digital stethoscopes), control units, video screens, network connections, network firewalls and telecommunication connections. Personnel need the technical skills to set up and maintain all of this equipment and to be instantly responsive when live examination sessions cause technical difficulties. Qualifications for the role include familiarity with digital communications, telecommunications services, and networks, as well as video and audio equipment with an emphasis on videoconferencing.

\section{The role of the Business Agent}

This role may or may not be assumed by the financial affairs group or the billing group within your organization. The principal duty is to manage the financial affairs of the program by the creation and maintenance of the necessary records system that facilitates timely and appropriate billing for services. Secondly, it involves tracking the requirements of the relevant payers regarding payments for telemedicine services and working to ensure that timely reimbursement payments are made. In alternative payment scenarios that involve contracts for services, the business agent takes responsibility for developing those contracts and complying with their financial terms. Qualifications for this role include familiarity with billing processes, a business background and experience with financial records systems.

\section{The role of the Specialty Coordinator}

This role is responsible for the actual conduct of the telemedicine consultations within a given specialty when there are enough patients to justify the assignment. The coordinator may take responsibility for two or more 
specialties. The typical specialty coordinator schedules the medical/clinical specialists for telemedicine consultations, schedules and verifies the presence of the patients to be seen, ensures that the necessary clinical records information is available for the patient, verifies that the equipment is functional prior to the beginning of consultations, assists the specialist as necessary during the consultation and completes any necessary paperwork subsequent to the consultation including billing forms and communications with referring physicians. Qualifications for the role include familiarity with medical/clinical terminology and the process of care, basic technical skills sufficient to operate the equipment to be used and the ability to troubleshoot problems during the course of a consultation.

\section{$N$. kind of supporting staff need at the remote sites}

To conduct high quality and successful telemedicine consultations at a remote site whether that is a hospital, clinic or some other facility, several roles need to be filled. These support staff serve as liaisons with the administration at remote sites, gather patient information, schedule consultations, conduct the clinical encounter and provide technology support. The people performing these roles must work closely with the site where the provider is located to carry out their responsibilities successfully.

\section{O. Telemedicine coordinator}

The telemedicine coordinator is the primary liaison with the provider sites. This person manages the telemedicine services at their own sites, including serving as the administrative point of contact, representing the program to the site's administration, medical/clinical staff and the local community, supervising local telemedicine personnel, arranging for appropriate space, supervising the scheduling of patients for telemedicine consultations, and interacting with local referring physicians as necessary to follow up on telemedicine consultations. This role typically also oversees the financial aspects of the telemedicine service at the remote site. Qualifications for the position include healthcare administrative experience as well as familiarity with and enthusiasm for telemedicine.

\section{$P$. The role of the local physician champion}

A local physician who will assume to role of medical/clinical director for telemedicine is critical to the successful operation of the program. This person's enthusiastic support of telemedicine provides credibility for the service at the local level and can often be persuasive in convincing other local physicians to refer their patients to telemedicine even if it means changing their referral patterns. At the same time, the existence of a local physician champion can convince the administration of the site that their telemedicine program is viable since at least one physician from the community supports it.

Copyright to IJARCCE

\section{$Q$. The telemedicine presenter}

A skilled telemedicine presenter is critical to the successful conduct of a telemedicine consultation. This person is the extended "eyes and ears" of the specialist at the other end of the connection during live, interactive consults. When the store-and-forward consults are used, this person is the one who is responsible for capturing the digital images or other information forms as well as the necessary clinical information to forward to the specialist. They must be familiar with the operational protocols (Protocol Module - Coming Soon), be skilled in clinical interactions with patients and be able to operate all the equipment used. They must be able to take directions from the specialist, interpret them in the light of the situation and report back in a clinically meaningful manner. In many instances, the "presenter" is present in the room with the patient when a consult occurs. However, for certain types of services, such as psychiatric therapy services, the presenter may set up the room and then leave the room to ensure patient confidentiality. Often, the presenter also takes responsibility for scheduling individual patients and assuring that they arrive in a timely manner for their appointments. This person must also be able to take verbal directions from the specialist regarding additional testing, recommended treatments and instructions to the referring physician. Finally, they need to update and maintain the local copy of the patient's medical record with information from the telemedicine consultation when appropriate. Qualifications for a telemedicine presenter include training as a healthcare professional, technical knowledge sufficient to operate the equipment, and a patient orientation to make the patient comfortable during the encounter.

\section{III.TELEMEDICINE SERVICE}

The technical support required at a remote site usually consists of local telecommunications and networking, information systems and videoconferencing equipment troubleshooting and maintenance. For live, interactive telemedicine, this support needs to be on-call during the entire duration of the session so that contact can be restored as soon as possible, if it is lost. The increasing size of distributed telemedicine systems creates problem of data integration, vendor lock-in and interoperability. Telemedicine center Setup has complex critical component and need proper planning. This paper explains the Telemedicine Station using computer communication methodology and hybrid computing are successfully setup a telemedicine base station of center. The proper planning is implemented for Telemedicine station using Grid, Cloud, Mobile, Cluster and Super computing technology and reduced $80 \%$ of maintenance cost for telemedicine centre. Cloud computing Technology supports increasing large number of Big data storage of medical and solve the problem of data integration. Grid computing technology supports telemedicine node allocation, monitoring and load balancing of telemedicine network. Mobile computing supports mobile telemedicine in ambulance, Home patients monitoring. Cluster and Super computing www.ijarcce.com 
supports maintenance of telemedicine work station as shown in figure1

\section{A. Qualifications}

Local videoconferencing support requires someone who is trained in the use of the equipment. This can be accomplished either through vendor training courses, training at the central site offering the service or working with the staff at another remote site. Beyond that, a base level understanding of telecommunications as well as digital video and audio is necessary.

\section{B. Type of Support Required}

Many telemedicine consultations benefit from the ability to exchange clinical information electronically with the specialist provider. This may range from simple email used to transmitting scanned documents to elaborate shared electronic health records. The usual degree of support required at the remote site is the support and maintenance of the equipment, such as a computer workstation to access this information.

\section{Telemedicine services}

Recruiting physician specialists to provide telemedicine consultations is not an easy task. There are a number of issues and concerns to address including:

- $\quad$ Those who have never used telemedicine are skeptical of its feasibility and effectiveness and are concerned about the potential loss of the doctor-patient relationship that is fostered in face-to-face or in-person care.

- $\quad$ Many specialists perceive their time as already fully booked and do not feel they have the availability to see patients via telemedicine.

- $\quad$ Some specialists view telemedicine consultations as less remunerative than office visits because they cannot perform more expensive procedures.

- Many physicians claim to "have heard" that telemedicine does not really work very well.

- Most physicians believe they will not be reimbursed for telemedicine services and that it is a volunteer or charitable activity.

Many physicians do not believe that their organization will be supportive of telemedicine consultations.[11 to 18] The Telemedicine concept of service as shown in Figure1. Telemedicine service using computer communication network and mobile as shown in Figure 2. Figure 3 shows Technical Telemedicine service experiments implemented using $3 \mathrm{G}$ Internet. The biomedical signals as shown in figure 4 .

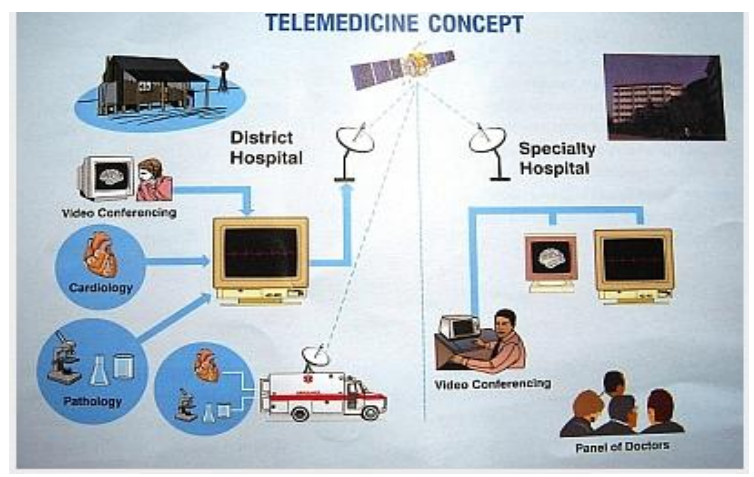

Fig. 1 Telemedicine concept

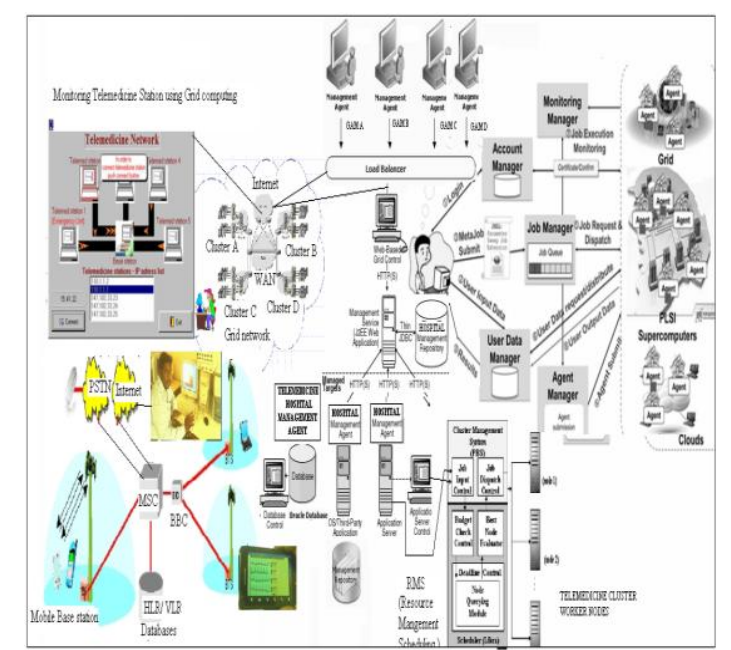

Fig. 2 Technical support of Telemedicine Service using Computer communication

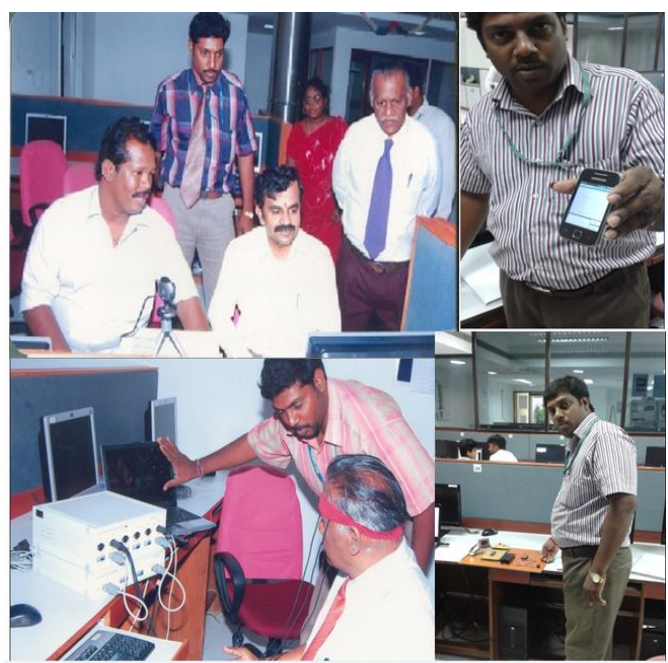

Fig. 3 Technical Experiments of Telemedicine service using computer and mobile in Rajiv Gandhi college of Engineering and technology,

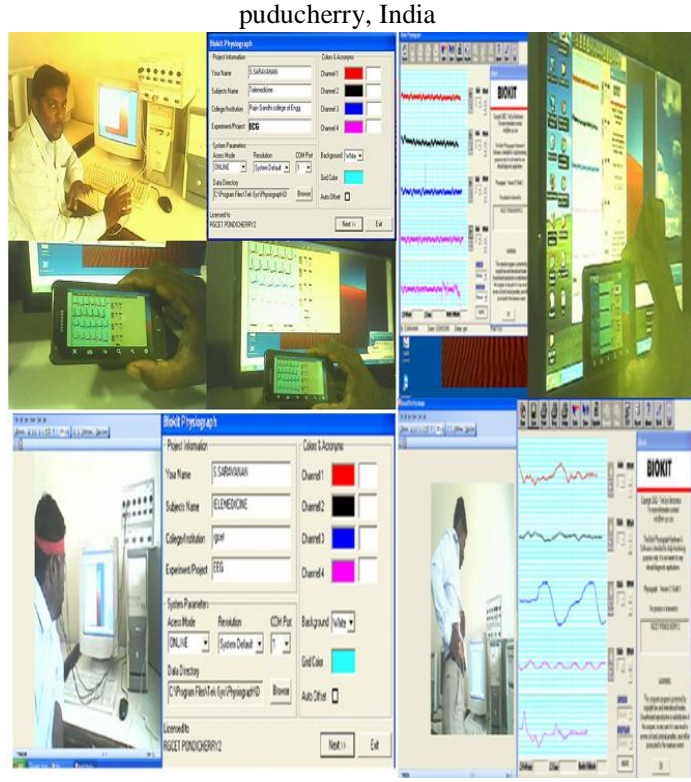

Fig. 4 Monitoring the Biomedical signals 


\section{IV.ASSURANCES OBTAINED REGARDING TELEMEDICINE TIME COMMITMENTS FROM DEPARTMENTAL ADMINISTRATORS}

The key to answering this question is the organization's top level commitment to telemedicine consult services. Departmental administrators are very sensitive to the direction of corporate strategies and how those strategies affect their department or service. To the extent that they clearly perceive that there is top level commitment to telemedicine and/or they see evidence of commitment in terms of resources allocated to telemedicine, they will be more likely to support those specialists who choose to participate in a telemedicine consultation service. Execution of a memorandum of understanding (MOU) with the department detailing the commitments they will allow assures that that there is a clear understanding among all participants and serves as a corporate memory when new administrators join the department. It is possible to achieve the same assurances through less formal arrangements, such as verbal agreements, corporate statements of intention to participate, or by building close professional relationships with the administrators through participation in administrative meetings. These all have the potential to work well but are more subject to reinterpretation and being forgotten over time.

\section{ConClusion}

ISRO supports $3 \mathrm{G}$ internets to telemedicine service to rural area using computer and Mobile communication. All the Indian people are satisfied by $3 \mathrm{G}$ internets for transmission of big data of patient from ambulance, hospital to specialist doctors. we thanks to ISRO health care government for $95 \%$ fulfilment.

\section{ACKNOWLEDGMENT}

Most thanks to computer science, Biomedical Departments of Head and management of Rajiv Gandhi college of Engineering and Technology in puducherry, India.

\section{REFERENCES}

[1] www.isro.org

[2] www.mit.gov.in

[3] Misra UK, Kalita J, Mishra SK, Yadav RR, "Telemedicine for distance education in Neurology- Preliminary experience in India",J Telemed Telecare, 2004;10(6):363-365

[4] Kartar Singh, Saroj K. Mishra, Ramnath Misra, Ratni

B.Gujral, Ramesh K.Gupta, Umakant Misra, Archana Ayyagari, BiswaN.Mohanty, Lily Kapoor L S Satyamurthy, B S Bhatia, A Bhaskaranarayana, RagesShah, "Satcom based Distance Education in Medicine - Evaluation of Orissa Telemedicine Network", Proceedings of $6^{\text {th }}$ IEEE Healthcom 2004, June 2004, Odawara, Japan

[5] Kartar Singh, Saroj K Mishra, Ramnath Misra, RatniB Gujral, Rakesh K Gupta, U K Misra, Archana Ayyagari, Rajesh Basnet, Biswanarayan Mohanty, "Strengthening Postgraduate Medical Education in Peripheral Medical Colleges through TM",Proceedings of $9^{\text {th }}$ Annual Conference of American Telemedicine Association, May 2004, Tempa, Florida, USA

[6] Misra UK, Kalita J, Mishra SK, Yadav RK, "Telemedicine in neurology: underutilized potential", Neurol India, 2005 Mar;53(1):27-31

[7] Telemedicine Application in Surgery : Indian Experience and Update on Telemedicine Activities in India, S.K.Mishra; ITU
(BDT) Q. $14 \quad 1 / 2$ Rapporteur's Group Meeting, June 2005,Tokyo,Japan

[8] Telemedicine: Experience at a Tertiary Care Hospital, S.K.Mishra; Proceedings of $7^{\text {th }}$ International Workshop on computing and networking in healthcare industry (Healthcom 2005), June 2005, Busan, Korea,

[9] Kapoor L, Mishra SK, Singh K, "Telemedicine: experience at SGPGIMS, Lucknow. J Postgrad Med. 2005Dec;51(4):312-5.

[10] Design and Implementation of Telemedicine Network in a Sub Himalayan State of India, Lily Kapoor, Rajesh Basnet, RepuDaman Chand, Sandeep Singh, Pankaj Joshi, Maneesh Semwal,Durgapal, K S Negi, Ragesh Shah,S K Mishra, Kartar Singh;Proceedings of 8th International Conference on e-Health Networking, Applications and Services, Healthcom 2006, ISBN: 1-4244-9704-5 (C) 2006 IEEE, P78-83 17-19th August 2006, New Delhi, India

[11] www.chennaionline.com/health/homearticles/2003/AI MS.asp

[12] www.tatamemorialcentre.com/misc/aboutus.htm

[13] www.hrudayalaya.com/Pages/Telemedicine.html

[14] wwwsankaranethralaya.org/tele_aim.htm

[15] www.aravind.org

[16] http://www.apollohospitals.com/medicalservices/ telemedicine.asp

[17] www.annauniv.edu/shortcourses/telehealth.doc

[18] http://www.mgmcri.ac.in/telemedicine.html

\section{BIOGRAPHIES}

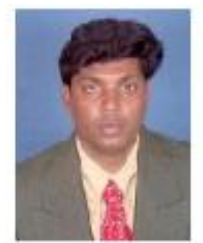

Mr. S.Saravanan, Asst. Professor( selection grade) in computer science department in Rajiv Gandhi college of Engineering and technology, puducherry, India. He has completed B.E ( Electronics and communication) in 1998, M.S (Information tech nology) in 2003, M.E ( computer science Engineering) in 2008, MBA(Education management) in 2010, M.Tech ( Communication System) in 2012,. He has 14 years experience in teaching of various Department of Electronics and communication, Biomedical Engineering and Computer science Engineering. He has teaching experience from 1998

Web site link:

http://www.rgcetpdy.ac.in/Profile/CSE/S.\%20Saravanan1.pdf

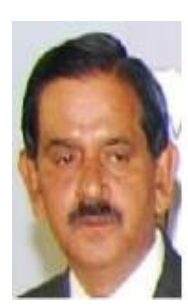

Dr. Sundaramurthy Reddy is working as a Professor in MBA Department from June 2008 in Rajiv Gandhi college of Engineering and Technology, Puducherry, India. He has finished MBA (Marketing \& System) and Phd in MBA ( Paradigm shift in pharma industry with reference to health care in puducherry city. $\mathrm{He}$ has experienced from 1998.

Web site link: http://www.rgcetpdy.ac.in/Profile/MBA/FORMAT\%20faculty\%20proforma\%20-\%20SSR.pdf 